\title{
Correction to: Associations between post- operative rehabilitation of hip fracture and outcomes: national database analysis
}

Bowen Su', Roger Newson' ${ }^{1}$, Harry Soljak² and Michael Soljak 3, ,* $^{2}$

\section{Correction}

Following publication of the original article [1], the author reported an error in the Title. The original article has been corrected. The details of the error are as follows:

Incorrect Title:

Associations between post-operative rehabilitation of hip fracture and outcomes: national database analysis (90 characters)

Correct Title:

Associations between post-operative rehabilitation of hip fracture and outcomes: national database analysis

\section{Author details}

${ }^{1}$ Department of Primary Care \& Public Health, School of Public Health, Imperial College London, W6 8RP, London, UK. ²Department of Anaesthetics, St Peter's Hospital, Chertsey KT16 OPZ, UK. ${ }^{3}$ Centre for Population Health Sciences (CePHaS), Lee Kong Chian School of Medicine, Nanyang

Technological University, Singapore 308232, Singapore.

Published online: 19 September 2018

\section{Reference}

1. Su B, et al. Associations between post-operative rehabilitation of hip

fracture and outcomes: national database analysis. BMC Musculoskelet Disord. 2018;19:211. https://doi.org/10.1186/s12891-018-2093-8.

\footnotetext{
*Correspondence: michael.soljak@ntu.edu.sg

${ }^{3}$ Centre for Population Health Sciences (CePHaS), Lee Kong Chian School of Medicine, Nanyang Technological University, Singapore 308232, Singapore ${ }^{1}$ Department of Primary Care \& Public Health, School of Public Health, Imperial College London, W6 8RP, London, UK
} 